\title{
Magnetic imaging with a Zernike-type phase plate in a transmission electron microscope
}

Pollard, Shawn; Malac, Marek; Beleggia, Marco; Kawasaki, Masahiro; Zhu, Yimei

Published in:

Applied Physics Letters

Link to article, DOI:

$10.1063 / 1.4803908$

Publication date:

2013

Document Version

Publisher's PDF, also known as Version of record

Link back to DTU Orbit

Citation (APA):

Pollard, S., Malac, M., Beleggia, M., Kawasaki, M., \& Zhu, Y. (2013). Magnetic imaging with a Zernike-type phase plate in a transmission electron microscope. Applied Physics Letters, 102(19), [192401].

https://doi.org/10.1063/1.4803908

\section{General rights}

Copyright and moral rights for the publications made accessible in the public portal are retained by the authors and/or other copyright owners and it is a condition of accessing publications that users recognise and abide by the legal requirements associated with these rights.

- Users may download and print one copy of any publication from the public portal for the purpose of private study or research.

- You may not further distribute the material or use it for any profit-making activity or commercial gain

- You may freely distribute the URL identifying the publication in the public portal 


\section{$1 D$ Applied Physics Letters \\ Magnetic imaging with a Zernike-type phase plate in a transmission electron microscope}

Shawn Pollard, Marek Malac, Marco Beleggia, Masahiro Kawasaki, and Yimei Zhu

Citation: Appl. Phys. Lett. 102, 192401 (2013); doi: 10.1063/1.4803908

View online: http://dx.doi.org/10.1063/1.4803908

View Table of Contents: http://apl.aip.org/resource/1/APPLAB/v102/i19

Published by the AIP Publishing LLC.

\section{Additional information on Appl. Phys. Lett.}

Journal Homepage: http://apl.aip.org/

Journal Information: http://apl.aip.org/about/about_the_journal

Top downloads: http://apl.aip.org/features/most_downloaded

Information for Authors: http://apl.aip.org/authors

\section{ADVERTISEMENT}



\title{
Magnetic imaging with a Zernike-type phase plate in a transmission electron microscope
}

\author{
Shawn Pollard, ${ }^{1,2}$ Marek Malac, ${ }^{3, a)}$ Marco Beleggia, ${ }^{4}$ Masahiro Kawasaki, ${ }^{5}$ and Yimei Zhu ${ }^{1,2}$ \\ ${ }^{1}$ Brookhaven National Laboratory, Department of Condensed Matter Physics and Materials Science, \\ Upton, New York 11973, USA \\ ${ }^{2}$ Department of Physics and Astronomy, Stony Brook University, Stony Brook, New York 11794, USA \\ ${ }^{3}$ National Institute for Nanotechnology, 11421 Saskatchewan Drive, Edmonton, Alberta T6G 2M9, Canada \\ ${ }^{4}$ Center for Electron Nanoscopy, Technical University of Denmark, $2800 \mathrm{Kgs}$. Lyngby, Denmark \\ ${ }^{5}$ JEOL Ltd., 1-2 Musashino, 3-chome, Akishima, Tokyo 196-8558, Japan
}

(Received 11 January 2013; accepted 19 March 2013; published online 13 May 2013)

\begin{abstract}
We demonstrate the use of a hole-free phase plate (HFPP) for magnetic imaging in transmission electron microscopy by mapping the domain structure in PrDyFeB samples. The HFPP, a Zernikelike imaging method, allows for detecting magnetic signals in-focus to correlate the sample crystal structure and defects with the local magnetization topography, and to evidence stray fields protruding from the sample. Experimental and simulated results are shown and are compared with conventional Fresnel (out-of-focus) images without a phase plate. A key advantage of HFPP imaging is that the technique is free from the reference wave distortion from long-range fields affecting electron holography. [http://dx.doi.org/10.1063/1.4803908]
\end{abstract}

Relating the electrostatic and magnetic potentials and fields with morphology, structure, defects, and orientation of individual nanoscale objects is a key prerequisite to understand the physics driving their utility in practical applications. A transmission electron microscope (TEM) allows detailed investigation of structure and composition at atomic resolution, but imaging slowly varying electrostatic and magnetic fields is notoriously difficult. Here, we describe the application of a "hole-free phase plate" (HFPP) in a TEM ${ }^{1-3}$ to image local ordering in a magnetic sample, as well as the external stray field generated at the edges of magnetic materials. The method can be equally well applied to imaging of electrostatic fields.

Phase objects such as long-range electrostatic and magnetic fields in vacuum are very difficult to detect with standard TEM imaging modes. ${ }^{4}$ While electron holography allows the quantification of slowly varying fields surrounding samples, problems arise from the disturbance of the reference wave by the investigated field itself $f^{5}$ and the method is experimentally challenging. ${ }^{6}$ On the other hand, Fresnel imaging is fairly straightforward, but uses high defocus, is only sensitive to large magnetization gradients (such as at domain boundaries) and results in loss of structural details at the nanoscale. ${ }^{7,8}$ The use of a $\mathrm{HFPP}^{8}$ reported here gives us the opportunity to study slowly varying magnetic fields at $<5 \mathrm{~nm}$ resolution, including the fields in vacuum surrounding the sample. Electrostatic and magnetic fields in vacuum are of great importance in fundamental research involving the interactions of various systems, such as arrays of nanomagnets, ${ }^{9}$ artificially induced domain wall pinning sites in nanowires, ${ }^{10-14}$ or ferroelectric nanoparticles. ${ }^{15}$ Similar to Foucault imaging, a HFPP can be used to visualize simultaneously the electromagnetic fields and the structure/defects of the sample at the nanoscale.

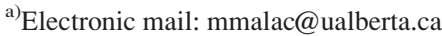

In order to compare images obtained using a HFPP with Fresnel images, data were acquired using a JEOL 2100 FMLM, which is equipped with an objective pole piece optimized for magnetic imaging of materials ${ }^{16}$ operated at $200 \mathrm{kV}$. The HFPP, a $10 \mathrm{~nm}$ thick amorphous carbon thin film, ${ }^{8}$ was placed at the back focal plane of the objective lens. The HFPP images were collected near Gaussian focus and, for comparison, Fresnel images were acquired from the same sample area. We used $(\mathrm{Pr}, \mathrm{Dy})_{2} \mathrm{Fe}_{14} \mathrm{~B}$, a hard magnet with $\mu_{0} M_{S}=1.4 \mathrm{~T}$, exhibiting a magnetic stripe domain configuration. Simulations were performed and compared with the experimental images, giving insight into the contrast mechanism and functionality of the HFPP.

Figure 1(a) shows a Fresnel image taken $120 \mu \mathrm{m}$ underfocus, revealing magnetic domain walls in a typical stripe domain configuration. The same area, imaged near Gaussian focus, is shown in Fig. 1(b) with the HFPP inserted. If the HFPP is removed while other imaging conditions are kept, the same area reveals no domain-wall contrast, as shown in the inset in Fig. 1(b). All domain walls can be identified at the same locations in both Fresnel and HFPP images, but are not present in the inset. Line profiles across the domain walls and in vacuum are shown in Figs. 1(c)-1(e). The HFPP image shows intensity variations in vacuum near the sample edge that are not present in Fig. 1(a), a result of stray magnetic field in vacuum. Additionally, it is apparent that the contrast varies depending on the distance from vacuum within the sample. This is explored later in this work in combination with simulations. Finally, the HFPP allows for clear determination of the sample edge location without the presence of contrast oscillations and delocalization typical in Fresnel images.

The inset of Fig. 1(b), showing a bright field image of the same area of the sample, allowed us to estimate the local thickness of the sample from the image intensity and the known collection angle of the microscope. ${ }^{17}$ The sample appears to be a wedge with about $11^{\circ}$ apex angle. The 

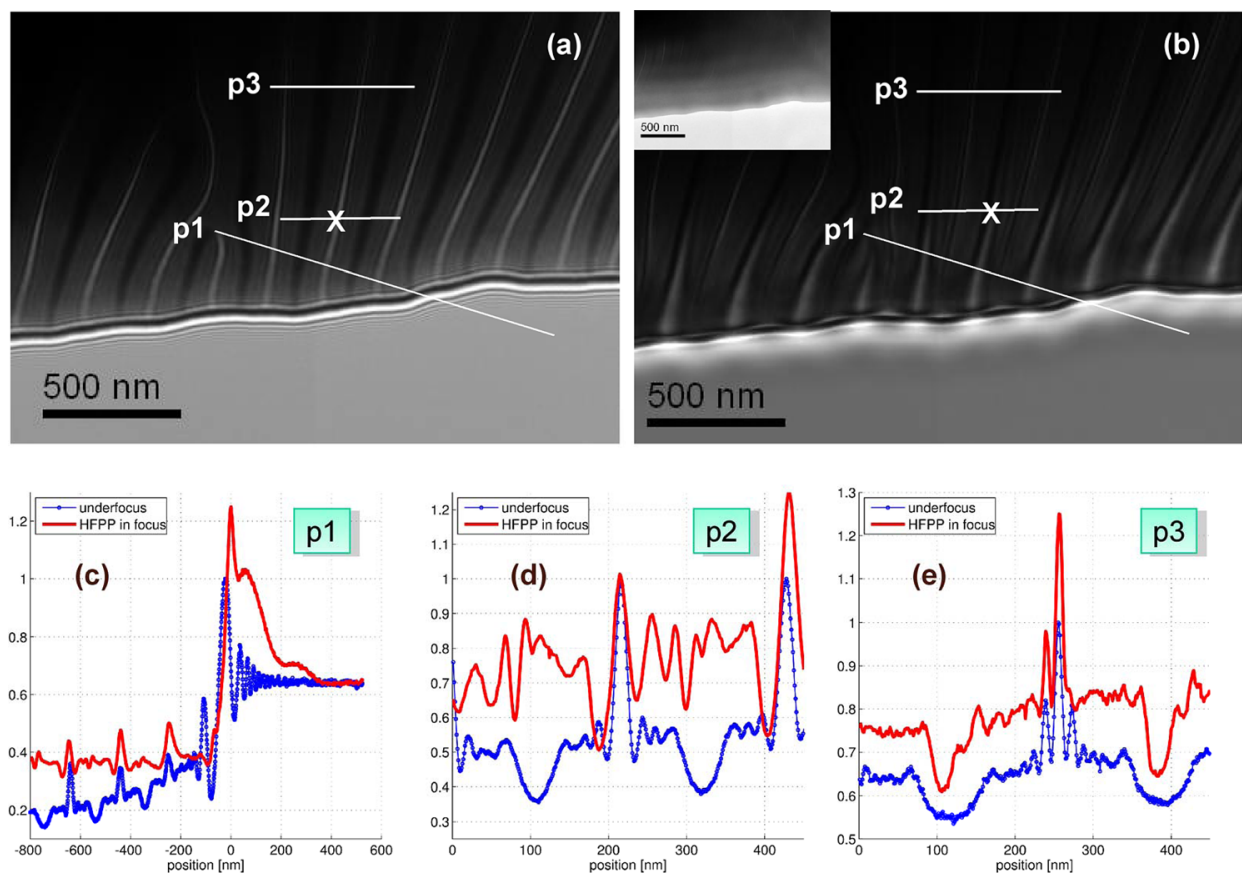

FIG. 1. Phase contrast images of the (Pr,Dy $)_{2} \mathrm{Fe}_{14} \mathrm{~B}$ magnetic stripe domain sample: (a) Fresnel (out-of-focus) image taken at $120 \mu \mathrm{m}$ underfocus. (b) HFPP image acquired infocus of the same area as in (a). The inset is an in focus image, also of the same area, without the HFPP. (c)-(e) Image intensity profiles taken along the lines p1-p3, respectively. The X in $\mathrm{p} 2$ marks a bright fringe used for alignment of HFPP p2 relative to Fresnel p2. Other profiles were aligned similarly. They were normalized to 1 and the HFPP profiles were offset by +0.25 for clarity. nominal magnetization is about $1.4 \mathrm{~T}$ for this material allowing us to compare the acquired images with simulations. To calibrate the collection semiangle, ${ }^{17}$ a $40 \mathrm{~nm}$ thick permalloy sample was imaged using the same conditions as in the inset of Figure 1(a).

Figure 2 allows us to evaluate the contrast transfer function (CTF) of the microscope in the HFPP mode. Figure 2(a) shows a logarithmic plot of the radially averaged intensity of the Fourier transform of an amorphous carbon sample. The red solid line is for standard imaging conditions of the TEM while the blue dashed-dotted curve is for HFPP imaging mode. The intensity shows improvement over the standard imaging at low spatial frequencies by several orders of magnitude. Figure 2(b) shows the phase shift obtained by locating the extrema in (a) and assigning a $\pi / 2$ phase shift to each interval between the extrema. ${ }^{8}$ The curves in Figures 2(a) and 2(b) indicate that the HFPP transfers information at much lower spatial frequencies than standard TEM imaging mode. The transfer of low spatial frequencies is responsible for visualization of the slowly varying magnetic field in vacuum near the edge of the specimen in Figure 1(b). The experimental data for HFPP and standard imaging mode were collected under the same imaging conditions. First, HFPP data were acquired. Then the HFPP was retracted from the beam path and standard TEM images of the same sample area under identical conditions were acquired.

The practical outcomes demonstrated by this work show that HFPP imaging can be used to visualize the magnetization topography of a magnetic sample as well as the stray fields in vacuum near the sample edge. Additionally, the lower degree of blurring in HFPP than in Fresnel imaging allows for simultaneous observations of sample morphology and microstructure (in this case exemplified by locating the sample edge without loss of detail). However, quantitative interpretation of the results can be difficult: complications arise from the dependence of the signal on a variety of different parameters, including in particular sample thickness and charge distribution on the phase plate. ${ }^{8}$

Aiming at achieving a semi-quantitative analysis, we perform image simulations by describing the effect of the phase plate with the transmission function,

$$
f(q)=\phi \operatorname{Re}\left[\frac{2}{\pi} E\left(\frac{q^{2}}{q_{c}^{2}}\right)-1\right],
$$

where Re denotes the real part, $E(x)$ is the complete elliptic integral of the second kind, $\phi$ is the phase shift established between the center and the edge of the illuminated area on the HFPP $^{8}$ (the parameter $\phi$ is the HFPP analogue of the Zernike phase shift in conventional phase plates), and $q_{c} \lambda, \lambda$ being the wavelength of incident electrons, is the angular size of the transmitted beam impinging on the HFPP. Equation (1) is added to the standard terms $\left(\Delta Z, C_{S}, C_{C}\right.$, etc.) in the aberration function of the microscope, ${ }^{4,8}$ and represents the phase shift induced by the charged HFPP at position $r=q \lambda L$, where $L$ is the camera length. Simulations were carried out using a Mathematica code developed by one of the authors (M.B.).

Profiles for both Fresnel (Fig. 3(a)) and HFPP (Fig. 3(b)) simulations and experimental images taken $800 \mathrm{~nm}$ from the sample edge (corresponding to p3 in Figure 1) are shown. To calculate the profiles, we modeled the phase shift due to the stripe domain pattern as a triangular wave with half periodicity equal to the average domain size $d$ and an amplitude of $\pi \phi_{0} \mu_{0} M_{s} t d$ where $\phi_{0}$ is the flux quantum and $t$ is the local thickness of the sample. Finally, we show a tableau of intensity profiles calculated as a function of sample thickness while maintaining a fixed HFPP potential (Fig. 3(c)). There are significant contrast changes in the HFPP image depending on the thickness, an important effect to consider when comparing simulations and experiments. Fig. 3(d) shows a sample experimental area near the edge of the specimen with increasing thickness away from vacuum, 

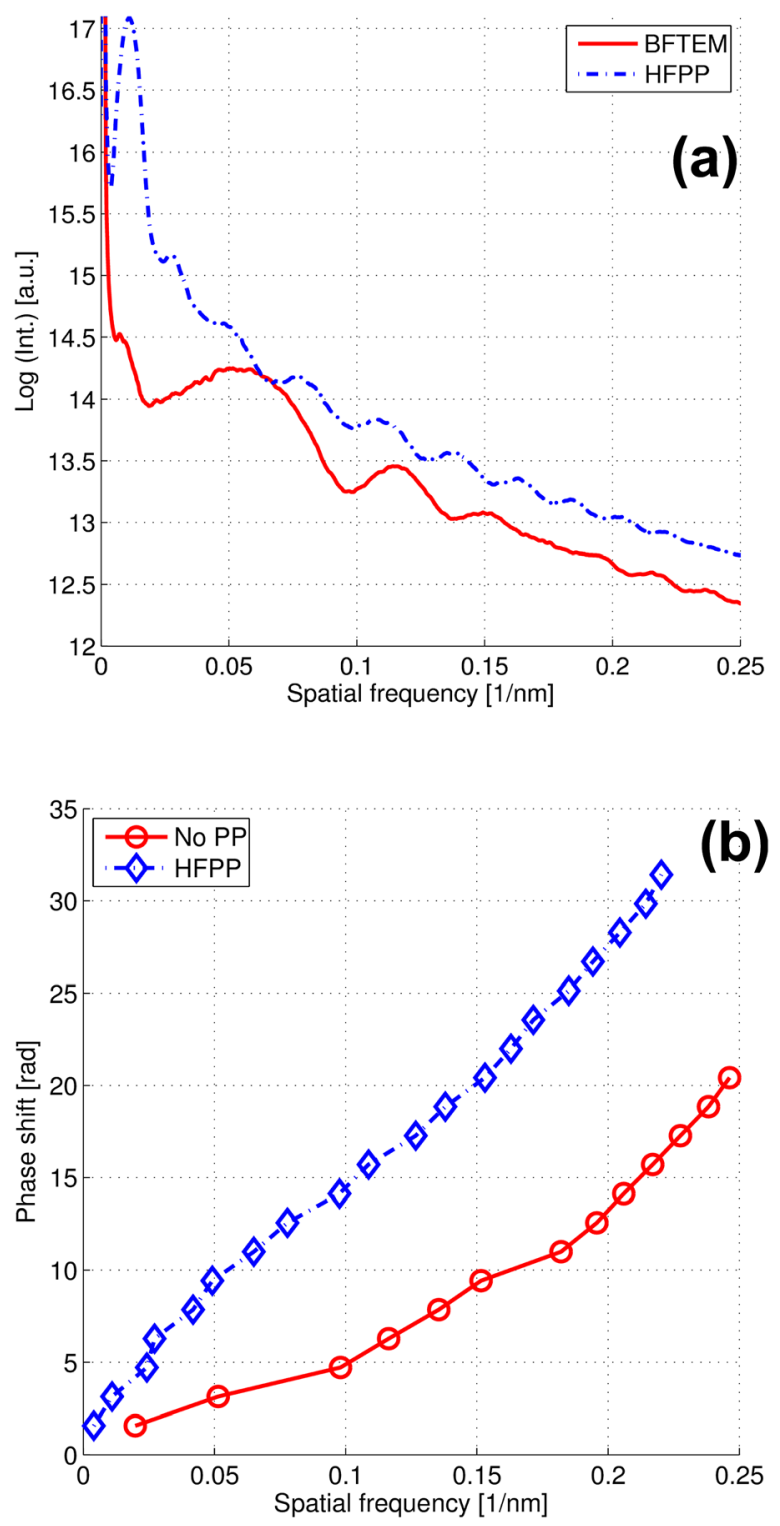

FIG. 2. (a) Intensity profile of the diffractogram from amorphous carbon sample, taken under the same imaging conditions as used in Figure 1, with the bright-field transmission electron microscopy (BFTEM) (Fresnel) and HFPP shown in red solid and blue dashed lines, respectively. The profiles are plotted with no vertical offset applied. (b) Phase shift (aberration function) $X(q)$ determined from the position of minima and maxima in (a). The HFPP transfer function (blue dashed line) exhibits more efficient transfer at the low frequencies, at $q$ from $4 \times 10^{-3}$ to $5 \times 10^{-2} \mathrm{~nm}^{-1}$, than the BFTEM (Fresnel) mode.

revealing a good qualitative agreement between simulations (Fig. 3(c)) and experiments (Fig. 3(d)). The apparent variations in the domain wall contrast with distance from vacuum (both intensity and the spread of the signal) are consistent with the thickness wedge measured earlier. However, the bright fringe along the domain wall close to the vacuum appears to broaden more in the experimental image than in the simulation. This is most likely due to the enlargement of the domain wall caused by demagnetizing fields near the edge, a factor that was not taken into account in the simulations. A better agreement could be obtained with the knowledge of the potential profile on the HFPP, and is one avenue for future work aimed at improving the HFPP technique towards quantitative interpretation. ${ }^{8}$
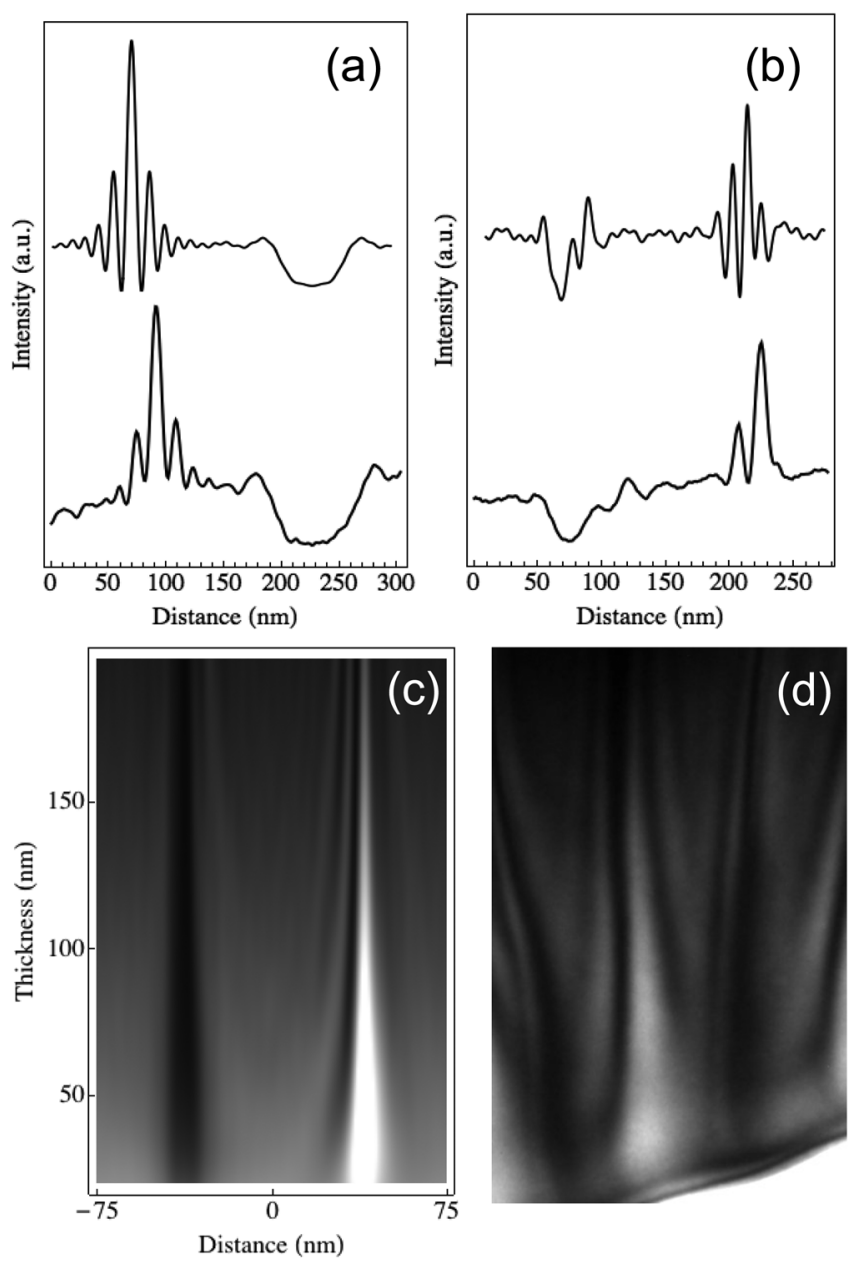

FIG. 3. Top: comparison between experimental (upper) and simulated (lower) intensity profiles taken at a distance $800 \mathrm{~nm}$ from the sample edge (where the thickness of the sample is approximately $200 \mathrm{~nm}$ ) for Fresnel (a) and HFPP (b) images. The intensity gradient visible in the experimental images is a result of the thickness slope of the sample. Bottom: (c) HFPP image intensity profile tableau computed with variable thickness and fixed HFPP shift $=\pi / 10$; (d) experimental image of a sample area with increasing thickness, showing both types of domain walls as in (c). The spread of the intensity near vacuum is seen in both images.

In summary, we have demonstrated the usefulness of a hole-free phase plate to image the domain structure and stray magnetic fields in vacuum in a $(\mathrm{Pr}, \mathrm{Dy})_{2} \mathrm{Fe}_{14} \mathrm{~B}$ magnetic thin film. The simplicity of the HFPP $\operatorname{method}^{8}$ compared to others currently available shows great promise for use in a variety of problems involving nanomagnetism and nanoferroelectricity, particularly where characterizing the stray field or simultaneously determining microstructure is important. For instance, it is well known that magnetic domain walls can pin at grain boundaries or other defects. ${ }^{18,19}$ However, determining the characteristics of the potential well generated by defects has proven difficult without being able to simultaneously image microstructure as well as the local magnetization. The HFPP represents a promising tool in answering questions related to the role of defects in magnetization processes since the sample features of interests are imaged with less distortion and contrast delocalization than the conventional phase contrast methods in Lorentz microscopy. ${ }^{8}$ While more work needs to be carried out in order to achieve quantitative capabilities of the HFPP method, the 
sensitivity to stray fields could potentially allow for magnetic information to be extracted from samples that are typically ill-suited for magnetic imaging, such as spin-valves that are too thick for direct probing with an electron beam.

Experimental work was carried out at the Department of Condensed Matter Physics and Material Science, Brookhaven National Laboratory, and was supported by the U.S. Department of Energy, Office of Basic Energy Science, Material Sciences and Engineering Division, under Contract No. DE-AC02-98CH10886. Support of National Research Council and NSERC in Canada is gratefully acknowledged.

${ }^{1}$ R. Danev and K. Nagayama, Ultramicroscopy 88, 243-252 (2001).

${ }^{2}$ M. Beleggia, Ultramicroscopy 108, 953 (2008).

${ }^{3}$ M. Malac, M. Beleggia, R. Egerton, and Y. Zhu, Ultramicroscopy 108, 126-140 (2008).

${ }^{4}$ L. Reimer, Transmission Electron Microscopy, 4th ed. (Springer-Verlag, 1997).

${ }^{5}$ G. Matteucci, G. F. Missiroli, and G. Pozzi, Adv. Imaging Electron Phys. 99, 171-240 (1997).

${ }^{6}$ A. Tonomura, J. Electron Microsc. 44, 425 (1995).
${ }^{7}$ J. Zweck, M. Schneider, M. Sessner, T. Uhlig, and M. Heumann, Adv. Solid State Phys. 41, 533-545 (2001).

${ }^{8}$ M. Malac, M. Beleggia, M. Kawasaki, P. Li, and R. F. Egerton, Ultramicroscopy 118, 77-89 (2012).

${ }^{9}$ C. Phatak, A. K. Petford-Long, O. Heinonen, M. Tanase, and M. De Graef, Phys. Rev. B 83, 174431 (2011).

${ }^{10}$ S.-M. Ahn, K.-W. Moon, C.-G. Cho, and S.-B. Choe, Nanotechnology 22, 085201 (2011).

${ }^{11}$ V. L. Mironov, O. L. Ermolaeva, E. V. Skorohodov, and A. Yu. Klimov, Phys. Rev. B 85, 144418 (2012).

${ }^{12}$ L. O'Brien, D. Petit, H. T. Zeng, E. R. Lewis, J. Sampaio, A. V. Jausovecand, D. E. Read, and R. P. Cowburn, Phys. Rev. Lett. 103, 077206 (2009).

${ }^{13}$ L. O'Brien, D. Petit, E. R. Lewis, R. P. Cowburn, D. E. Read, J. Sampaio, H. T. Zeng, and A.-V. Jausovec, Phys. Rev. Lett. 106, 087204 (2011).

${ }^{14}$ L. O'Brien, A. Beguivin, A. Fernandez-Pacheco, D. Petit, D. E. Read, and R. P. Cowburn, Appl. Phys. Lett. 101, 062415 (2012).

${ }^{15}$ M. J. Polking, M.-G. Han, A. Yourdkhani, V. Petkov, C. F. Kisielowski, V. V. Volkov, Y. Zhu, G. Caruntu, P. A. Alivisatos, and R. Ramamoorthy, Nature Mater. 11, 700-709 (2012).

${ }^{16}$ M. A. Schofield, M. Beleggia, Y. Zhu, and G. Pozzi, Ultramicroscopy 108, 625-634 (2008).

${ }^{17}$ H.-R. Zhang, R. F. Egerton, and M. Malac, Micron 43, 8-15 (2012).

${ }^{18}$ R. L. Compton and P. A. Crowell, Phys. Rev. Lett. 97, 137202 (2006).

${ }^{19}$ T. Uhlig, M. Rahm, C. Dietrich, R. Hollinger, M. Heumann, D. Weiss, and J. Zweck, Phys. Rev. Lett. 95, 237205 (2005). 